\title{
ANÁLISE DA QUALIDADE DE VIDA NO TRABALHO NO SETOR DE TELECOMUNICAÇÕES BRASILEIRO
}

\section{ANALYSIS OF QUALITY OF WORK LIFE IN THE BRAZILIAN TELECOMMUNICATIONS SECTOR}

\author{
Oswaldo Luiz Agostinho', Jefferson de Souza Pinto², \& Randal Victor Gibbin ${ }^{3}$ \\ ${ }^{12}$ Faculdade de Engenharia Mecânica da Universidade Estadual de Campinas - FEM/UNICAMP. \\ ${ }^{1}$ agostinh@fem.unicamp.br ${ }^{2}$ jeffsouzap@gmail.com ${ }^{3 *}$ rgibbin@ criteriologico.com.br
}

\section{ARTIGO INFO.}

Recebido em: 23.03.2021

Aprovado em: 15.04.2021

Disponibilizado em: 03.05.2021

\section{Palavras-chave:}

Responsabilidade social corporativa; qualidade de vida no trabalho; análise estatística; análise de corpus de texto; setor de telecomunicações.

KEYWORDS:

Corporate social responsibility; quality of work life; statistical analysis; text corpus analysis; telecommunications sector.

*Autor Correspondente: Gibbin, R. V.

\section{RESUMO}

Contexto: A Qualidade de Vida no Trabalho (QVT) assumiu lugar de destaque nos últimos anos como componente da cultura organizacional, sendo um dos pilares da sustentabilidade.

Objetivo: Identificar como os quatro maiores grupos de telecomunicações atuantes no Brasil compreendem a QVT neste contexto (dois dos quais listados na carteira ISEB3/2020).

Método: Procedeu-se à análise textual (lexical), processada pelo software IRAMUTEQ, da documentação de sustentabilidade disponível nos repositórios públicos destas organizações, desdobrada na Análise Lexicográfica, Classificação Hierárquica Descendente e Análise de Similitude.

Resultados: Os resultados evidenciam uma prevalência de práticas vinculadas à saúde ocupacional, capacitação, desenvolvimento e voluntariado.

Conclusão: Verificam-se oportunidades de aprimoramento em questões ligadas à redução de estresse, mentoria, resolução de conflitos, premiação e reconhecimento. Esta pesquisa tem sua originalidade no uso da técnica de análise textual ao objeto de estudo.

\begin{abstract}
Context: Quality of Work Life (QWL) has assumed a prominent place in recent years as a component of organizational culture, being one of the pillars of sustainability.

Objective: Identify how the four largest telecommunications groups operating in Brazil understand $Q W L$ in this context (two of which listed in the ISEB3/2020 portfolio).

Method: A lexical analysis was carried out, processed by the IRAMUTEQ software, of the sustainability documentation available in the public repositories of these organizations, deployed in the Lexicographic Analysis, Descending Hierarchical Classification and Similitude Analysis.

Results: The results show a prevalence of practices related to occupational health, training, development, and volunteering.

Conclusion: Opportunities for improvement are verified in issues related to stress reduction, mentoring, conflict resolution, awards, and recognition. This research has its originality in the use of the textual analysis technique to the object of study.
\end{abstract}




\section{INTRODUÇÃO}

O desenvolvimento sustentável apresenta desdobramentos nas esferas econômica, ambiental e social, possui forte relação com os meios empregados para a criação de riqueza, podendo ser entendida como o suprimento das necessidades do indivíduo, sem comprometer as futuras gerações (World Commission on Environment and Development [WCED], 1987; Sakar \& Searcy, 2016).

Contribuindo para a relevância do tema, tem-se a emergência do termo Responsabilidade Social Corporativa (RSC) dentro da comunidade acadêmica e empresarial, particularmente na primeira década deste século, assumindo desdobramentos táticos e estratégicos nas organizações (Bansal, Jiang, \& Jung, 2015).

A criação de programas de certificação, como da norma SA 8000 (Social Accountability) em 1997, visando criar um modelo de gestão de responsabilidade social, assim como da série ISO 14000 em 1996, procurando estabelecer um sistema de gestão ambiental, corroboraram para tais desdobramentos, os quais transcendem a organização, chegando à cadeia de suprimentos (Bizzo \& Andrade, 2018; Ueasangkomsate, 2019; International Standartization Organization [ISO], 2020).

Destaca-se também no período publicações de relatórios de saúde, segurança e sustentabilidade por parte de empresas europeias, o que fomentou a criação de programas de certificação e premiações. Surge em 1997 a Global Report Initiative (GRI) a fim de criar padrões de reporte de ações sob a concepção do Triple Bottom Line (TBL) (Milne, Tregidga, \& Walton, 2003; Global Report Iniciative [GRI], 2020).

No Brasil é criado em 2005 o Índice de Sustentabilidade Empresarial (ISE) pela B3 (Brasil, Bolsa, \& Balcão), empresa de infraestrutura de mercado financeiro, com o intuito de servir como uma ferramenta de benchmarking de ações de sustentabilidade corporativa, com pilares na eficiência econômica, respeito ao meio ambiente, justiça social e governança (Índice de Sustentabilidade Empresarial [ISE], 2020).

Anualmente a B3 define uma carteira de empresas certificadas como organizações que praticam ações de sustentabilidade empresarial, servindo para orientação a investidores (ISE, 2020).

Paralelamente, surge nos anos 70 o conceito de Qualidade de Vida no Trabalho (QVT), entendido como a adoção de um conjunto de ações a fim de melhorar a gestão, tecnologia e estrutura no ambiente de trabalho da organização, abrangendo conceitos pertinentes à economia, saúde, ecologia, ergonomia, psicologia, sociologia, administração e engenharia (Robie, Rosini, \& Oliveira, 2016).

Assim, tem-se que a QVT como um elemento integrante do construto do TBL, visto que trata da humanização do local de trabalho, materializada em iniciativas de preenchimento das necessidades fisiológicas, psicológicas, de bem-estar, assim como do controle do nível de estresse do trabalhador (Sierdovski, Stefano, \& Andrade, 2020).

Neste contexto, são propostas as questões de pesquisa (QP):

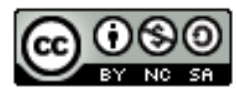



telecomunicações brasileiro. Brazilian Journal of Production Engineering, 7(2), 31-47.

QP1: Quais são as principais práticas de QVT adotadas pelas organizações objeto de estudo?

QP2: Como estas práticas estão relacionadas ao referencial teórico da QVT?

QP3: Existem aspectos do referencial teórico da QVT não praticados?

A fim de responder as questões de pesquisa, este trabalho tem o objetivo de identificar como os quatro principais grupos de telecomunicações atuantes no Brasil compreendem a qualidade de vida no trabalho, inserida no contexto da sustentabilidade, mediante procedimento de análise textual dos relatórios de sustentabilidade vigentes e disponíveis nos seus repositórios institucionais.

Este objetivo geral é suportado pelos seguintes objetivos secundários:

a) Pesquisar na literatura os conceitos de sustentabilidade, responsabilidade social corporativa e qualidade de vida no trabalho;

b) Avaliar, mediante procedimentos de análise lexicográfica clássica e multivariada (classificação hierárquica descendente e análise de similitude) como as organizações estudadas praticam ações de QVT;

c) Identificar aderências e oportunidades de aplicação das práticas de QVT em relação ao referencial teórico.

Este estudo se justifica pela relevância que a QVT assumiu nos últimos anos como componente da cultura organizacional nas empresas brasileiras, a exemplo da emergência de programas de capacitação de colaboradores, ações de voluntariado, estabelecimento de critérios transparentes para promoção e carreira e cuidados adotados para um melhor ambiente de trabalho (Jhunior \& Vilela, 2018; Sierdovski et al., 2020).

A definição do setor de telecomunicações como objeto de estudo ocorre pela pujança e tamanho, dentro do cenário empresarial brasileiro, contabilizando ao final de dezembro de 2019 mais de 300 milhões de acessos, entre telefonia móvel e fixa, acesso à internet banda larga fixa e serviços de TV por assinatura (Agência Nacional de Telecomunicações [ANATEL], 2019).

A seleção dos quatro maiores grupos decorre da representatividade que apresentam em relação ao total de acessos do setor, atingindo a marca de 279 milhões, correspondendo a 93\% do mercado brasileiro, destaca-se que dois destes grupos estão listados na carteira ISE B3/2020, como empresas comprometidas com a sustentabilidade (Agência Nacional de Telecomunicações [ANATEL], 2020a; ISE, 2020).

\section{FUNDAMENTAÇÃO TEÓRICA}

\subsection{Sustentabilidade}

O uso não controlado dos recursos naturais tem levado, nas últimas décadas, a uma crescente preocupação com a sustentabilidade; destaca-se o trabalho desenvolvido em 1987 pela Comissão Mundial sobre Meio Ambiente e Desenvolvimento (CMMAD) da Organização das Nações Unidas, consolidado no relatório Nosso Futuro Comum (Our Common Future) conceituando o termo, apontando problemas e indicando alternativas para tratar a questão ambiental em nível global e nacional (WCED, 1987). 

telecomunicações brasileiro. Brazilian Journal of Production Engineering, 7(2), 31-47.

Assim, segundo WCED (1987), o desenvolvimento sustentável implica “[...] em um processo de mudança no qual a exploração dos recursos, o direcionamento dos investimentos, a orientação do desenvolvimento tecnológico e as mudanças institucionais são feitos de forma coerente com as necessidades do futuro, assim como do presente [...]” ( p.17).

WCED (1987) define sustentabilidade como o ato de "[...] satisfazer as necessidades e aspirações do presente sem comprometer a capacidade das gerações futuras [...]” (p. 39).

No entanto, o termo sustentabilidade, quando aplicado ao contexto das organizações, apresenta diferentes significados, passando por uma evolução, desde a publicação seminal de Bowen, Social Responsibilities of the Businessmen (1953), até os dias atuais (Bowen, 1953; Sakar \& Searcy, 2016).

No início da segunda metade do século XX, o termo estava circunscrito a conciliação do lucro com interesses dos indivíduos e comunidade, atualmente adiciona questões de ética nos negócios, indo além do cumprimento de normas e legislações (Sakar \& Searcy, 2016).

Nesta evolução, destaca-se o conceito Triple Bottom Line (TBL); neste construto, uma organização competitivamente sustentável seria aquela que apresenta, além do bom desempenho financeiro, um uso racional dos recursos e resíduos e transparência nas relações com as partes influenciadas (stakeholders) (Elkington, 1998; Shulz \& Flanigan, 2016; Mascarenhas \& Barbosa, 2019).

No contexto organizacional, a sustentabilidade pode ser entendida como a prática de um conjunto de princípios que visam a gestão coerente dos negócios para um crescimento sustentável, inclui políticas e práticas éticas, uso racional dos recursos e lucros sustentáveis (Shulz \& Flanigan, 2016).

A visão de equilíbrio entre os fatores de ordem financeira (lucros), ambiental (uso racional dos recursos e descarte responsável) e social (gestão ética e transparente) foi introduzida por Elkington (1998) por meio do conceito de Triple Botton Line (TBL), alertando à época o "prenúncio de uma nova era", no qual as organizações precisarão exercer com maior cuidado suas relações com os stakeholders.

$\mathrm{Na}$ última década, o conceito TBL se evidencia em frameworks a fim de avaliar o desempenho organizacional, desdobrados em métricas financeiras, como lucro bruto e retorno sobre o investimento, ambientais como volume de resíduos tratados e consumo de carbono e sociais como grau de instrução e treinamento, investimento em projetos sociais e bem-estar do colaborador (Shulz \& Flanigan, 2016).

A necessidade em estabelecer padrões para a demonstração das ações de sustentabilidade levou em 1997 ao surgimento do GRI (Global Report Initiative), uma organização independente que, além de criar padrões e diretrizes para o desenvolvimento sustentável, busca promover a transparência nas relações entre os governos, sociedade e empresas (GRI, 2020).

Um exemplo nacional de framework para avaliar a sustentabilidade é o ISE (Índice de Sustentabilidade Empresarial), criado em 2005 pelo Centro de Estudos em Sustentabilidade da Fundação Getúlio Vargas (GVces), como resposta à demanda do mercado financeiro por 

telecomunicações brasileiro. Brazilian Journal of Production Engineering, 7(2), 31-47.

formas de acreditação em linha com os Princípios de Investimento Responsável (Principles of Responsible Investment - PRI) da Organização das Nações Unidas (Orsato, Garcia, Silva, Simonetti, \& Monzoni, 2015).

\subsection{Responsabilidade Social Corporativa (RSC)}

Bansal, Jiang e Jung (2015) descrevem empresas socialmente responsáveis como aquelas que procuram responder, no escopo de suas atividades, às necessidades e desejos dos stakeholders, compreendidos em acionistas, clientes, colaboradores, fornecedores, governo e comunidade.

Madorran e Garcia (2014) e Fernández, Bertin e Pineaur (2014) sustentam que a RSC é fonte de vantagem competitiva, por seus desdobramentos na gestão, percepção da marca, reputação e lealdade do consumidor.

Hernandez, Vazquez, Barcik e Dziwinski (2016) detalham a responsabilidade social em duas dimensões; a primeira externa, voltada para as relações com a sociedade e stakeholders externos; a segunda interna, com ênfase nos empregados. Acrescentam o enfoque interno tem fortes impactos na vantagem competitiva, visto que está ligado à mobilização do know-how acumulado da força de trabalho.

Kim, Milliman e Lucas (2020) destacam que a RSC participa na construção da imagem da empresa pelos seus empregados; esta visão da organização se desdobra em seus comportamentos e atitudes, como consequência da melhoria da autoestima e construção de identidade.

Ainda sob o ponto de vista do empregado observa-se que a RSC é um elemento na construção do sentido do trabalho, fator chave para o engajamento, pois traz o sentido de utilidade, significado e valor, em outras palavras, que seu trabalho faz diferença no seu contexto (Nazir \& Islam, 2020).

Em estudo conduzido em 590 pequenas e médias empresas, Hernandez, Vazques, Barcik e Dziwinski (2016) identificaram cinco eixos principais de ações de responsabilidade social: práticas responsáveis de recursos humanos, cultura organizacional, promoção de projetos sociais, políticas de compensação e qualidade de vida no trabalho.

Singhapakdi, Lee, Sirgy e Senasu (2015) destacam que, entre os domínios da RSC, o contexto do funcionário tem especial importância, materializado em ações de adequação do ambiente de trabalho, política de remuneração justa, fomento à diversidade e suporte familiar.

Adicionalmente, Sierdovski, Stefano e Andrade (2020) destacam que o desenvolvimento da qualidade de vida no trabalho demanda a priorização do ambiente laboral e o estabelecimento de critérios para a humanização, conforto, salário justo e instalações adequadas, a fim de prover a elevação da moral dos colaboradores e engajamento.

\subsection{Qualidade de Vida no Trabalho (QVT)}

A qualidade de vida no trabalho (QVT) é um conceito amplo relacionado com a satisfação do indivíduo como colaborador em uma organização, inclui aspectos sociais, de autoestima, estéticos e pertinentes ao conhecimento (Kim, Milliman, \& Lucas, 2020). 
Citação (APA): Agostinho, O. L., Pinto, J. de S., \& Gibbin, R. V. (2021). Análise da qualidade de vida no trabalho no setor de telecomunicações brasileiro. Brazilian Journal of Production Engineering, 7(2), 31-47.

Wardani e Anwar (2019) destacam que a adoção de práticas de QVT apresentam relação positiva com o engajamento do colaborador, elemento essencial para o aumento da produtividade, lucratividade, satisfação do cliente e pedidos de desligamento (turnover).

Os autores apontam que este engajamento tem forte relação com a potencialização do capital psicológico do indivíduo, ou seja, sua capacidade de transpor obstáculos, criar uma visão positiva do futuro e mostrar-se resiliente.

Qamari, Ferdinand, Dwiatmadja e Yuniawan (2020) dispõem que a QVT tem relação com elementos de ordem afetiva do empregado, mais especificamente, no alinhamento entre cultura e metas empresariais e os desejos e necessidades pessoais.

A QVT tem papel relevante no estabelecimento de relações laborais positivas, visto que o trabalho é fundamental para o desenvolvimento pessoal, sendo parte do ciclo de vida do colaborador, assumindo um aspecto fundamental no desenvolvimento integral do indivíduo (Mascarenhas \& Barbosa, 2019; Bustamante, Álvarez, Villalobos, \& Lucero, 2020).

Esta definição está em linha com Sirgy, Reilly, Wu e Efraty (2008), na qual a QVT está intimamente ligada à qualidade de vida do indivíduo, nos seus contextos profissional e pessoal, devendo atender às demandas que se originam na sua própria identidade, estruturada nos seus papéis, disposições afetivas, tendências comportamentais, valores e crenças.

Em resposta à estas demandas, a QVT engloba elementos de ordem física, cognitiva e emocional. Mais especificamente, fornece recursos adequados para o cumprimento das expectativas do colaborador, contribui para a redução de conflitos, aprimora os papéis atuais e desenvolve novos, reduz o estresse com o trabalho e aumenta o valor ou importância dos papéis desempenhados. (Sirgy et al., 2008; Kim et al., 2020)

De acordo com Sirgy et al. (2008), as políticas e programas de QVT podem ser classificados em 4 grandes grupos:

1) Ligados ao ambiente de trabalho: implantação de estruturas organizacionais descentralizadas, fomento ao trabalho em times, implantação de círculos da qualidade e promoção da cultura e ética da organização;

2) Ligados aspectos do cargo: participação na tomada de decisão em projetos de alto impacto e implantação de programas de capacitação/desenvolvimento de habilidades;

3) Ligados à gestão e supervisão das tarefas e responsabilidades: criação e viabilização de programas de feedback estruturado; supervisão sobre questões de ordem ética e comportamental (mentoria) e programas de gestão da qualidade total;

4) Ligados às políticas organizacionais de remuneração e promoção: criação e implantação de política de preenchimento de cargos com talentos internos (job posting) e planos de incentivo, ligados às realizações individuais, em times ou participação nos lucros.

Nos últimos anos, programas de QVT procuraram reduzir os conflitos entre o trabalho e a família, a fim de ajudar o colaborador no balanço das demandas nestes dois contextos. Surgem assim novos arranjos do trabalho, como o trabalho em casa (regime de home office), jornada flexível e semana de trabalho reduzida (Sirgy et al., 2008; Sierdovski et al., 2020). 

telecomunicações brasileiro. Brazilian Journal of Production Engineering, 7(2), 31-47.

A avaliação da QVT foi originalmente sugerida por Sirgy, Efraty, Siegel e Lee (2001), em um modelo de autoavaliação que, por meio de uma escala Likert, diagnostica o grau de preenchimento das necessidades do colaborador sob 7 aspectos, a saber:

1) Necessidades de saúde e segurança;

2) Necessidades econômicas e familiares;

3) Necessidades sociais;

4) Necessidades de reconhecimento;

5) Necessidades de atualização:

6) Necessidades de conhecimento;

7) Necessidades de criatividade.

Sinval, Sirgy, Lee e Marôco (2019), em pesquisa conduzida com brasileiros e portugueses no período de 2015 a 2017, fez uso deste modelo de avaliação, diagnosticando as percepções dos trabalhadores quanto à QVT, apontando nos colaboradores brasileiros níveis mais altos de satisfação no preenchimento das necessidades sociais, de conhecimento e de criatividade.

Alternativamente, Sierdovski et al. (2020), em estudo visando diagnosticar as práticas de QVT em empresas brasileiras, propõem um modelo de construto para o pilar social da sustentabilidade estruturado em um conjunto de 10 indicadores de QVT, os quais são:

1) Lazer e convívio social;

2) Satisfação no trabalho;

3) Remuneração justa;

4) Integração social;

5) Realização profissional e pessoal;

6) Comprometimento com a empresa, intenção de sair, taxa de rotatividade;

7) Personalidade e estresse no trabalho;

8) Saúde física e mental;

9) Condições de trabalho seguras e saudáveis;

10) Distribuição de salários, premiações e reconhecimento.

A pesquisa foi conduzida com mais de 60 mil indivíduos, no ano de 2016, demonstrando forte relação entre o modelo teórico de QVT proposto e práticas de avaliação de desempenho e feedback, programas de desenvolvimento de carreira e política de remuneração adequada e bonificação por resultados.

Mariappanadar (2020) destaca que, para o estabelecimento das práticas de QVT, a organização deve entender o contexto do seu colaborador de forma holística, ou seja, no trabalho e na família, a fim de obter seu engajamento. Complementa que a percepção do colaborador que a organização se preocupa com seu bem-estar tem papel na motivação para o trabalho e redução do estresse.

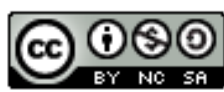



telecomunicações brasileiro. Brazilian Journal of Production Engineering, 7(2), 31-47.

Qamari et al. (2020) reforçam esta constatação e destacam que, em um ambiente em constante mudança, as práticas de QVT assumem um papel crucial para o sucesso das iniciativas estratégicas organizacionais, visto que implicam em prover aos empregados a oportunidade de decidirem sobre seu trabalho, seu ambiente laboral e até no desenvolvimento de novos produtos e serviços.

Também relacionam a QVT diretamente com a teoria de motivação de Maslow (1943) classificando suas práticas em dois níveis: práticas em resposta às necessidades mais básicas (saúde, segurança e de ordem econômica) e práticas em resposta às necessidades de alto nível (sociais, de autoestima e autorrealização).

\section{METODOLOGIA}

Segundo Prodanov e Freitas (2013), este trabalho se caracteriza como aplicado quanto à sua natureza e descritivo, no que se refere aos objetivos, visto que busca descrever o fenômeno da QVT no contexto da sustentabilidade nas organizações estudadas.

Sob a ótica do procedimento técnico, caracteriza-se como documental, visto que foram acessados os relatórios de sustentabilidade disponíveis nos repositórios de acesso público das organizações, dos quais foram extraídos corpus de texto para análise pelo software IRAMUTEQ.

Por fim, quanto à abordagem do problema, é classificado como qualitativo, visto que, a partir do corpus de texto, composto por extratos dos relatórios de sustentabilidade, foram realizadas análises estatísticas, a começar por análises lexicográficas, e posteriormente multivariadas, caso da Classificação Hierárquica Descendente (CHD) e Análise de Similitude (AS) (Lahlou, 2011; Camargo \& Justo, 2013).

Camargo e Justo (2013) definem a análise lexicográfica clássica como o processo de identificação e reformatação dos fragmentos de texto, a fim de calcular a quantidade de vocábulos, frequência média e hapax (palavras com frequência unitária).

Esta análise trabalha as palavras nas suas formas reduzidas (lematizadas) e gera um dicionário de formas ativas (ex: substantivos, verbos e adjetivos) e suplementares (ex: advérbios, numerais, artigos, conjunções e onomatopeias), para tanto faz-se necessário um aproveitamento mínimo de $70 \%$ do total de segmentos de texto (ST) do corpus original (Camargo \& Justo, 2013; Mendes, Tonin, Buzzi, \& Pontarolo, 2019).

A representação em nuvens de palavras é uma forma alternativa de organização das palavras em função da frequência e permite uma rápida identificação das palavras-chave do corpus (Mendes, Zangão, Gemito, \& Serra, 2016).

A Classificação Hierárquica Descendente (CHD) é uma análise multivariada que agrupa as palavras em classes de similaridade léxica, considerando a frequência e o posicionamento no texto, mediante o método Reinert. (Damart \& Ledunois, 2017; Mendes et al., 2019).

Esta análise permite a criação de uma estrutura analítica do texto, ou dendograma de classes, com os vocábulos agrupados em classes de semelhança (contexto semântico) pelo critério do 

telecomunicações brasileiro. Brazilian Journal of Production Engineering, 7(2), 31-47.

teste qui-quadrado de Pearson (X2), apresentando valores acima de 3,85 (Camargo \& Justo, 2013; Carvalho, Mota, Saab, 2020; Goulart, Viana, \& Cheung, 2020).

A Análise de Similitude (AS), também conhecida como análise de correspondência, baseia-se na teoria dos grafos, permite a identificação do grau de conexão das palavras (conexidade), mediante verificação das coocorrências, oferece uma visão da estrutura do corpus de texto em termos de representação cognitiva, representada usualmente em diagrama na forma de árvore (Camargo \& Justo, 2013; Mendes et al., 2016; Goulart et al., 2020).

Foram consultados os repositórios públicos (disponíveis nos sítios de relações com os investidores) dos quatro maiores grupos de telecomunicações atuantes no Brasil, denominadas TELECOM_1, TELECOM_2, TELECOM_3 e TELECOM_4.

Dos repositórios consultados, foram analisados os relatórios de administração e sustentabilidade disponíveis em sua última versão depositada (Quadro 1).

Quadro 1. Tipos de relatórios e versões consultadas na pesquisa.

\begin{tabular}{|c|c|c|}
\hline Organização & Tipo de Documento & Versão \\
\hline TELECOM_1 & Relatório de Sustentabilidade & 2017 \\
\hline TELECOM_2 & Relatório de Sustentabilidade & 2019 \\
\hline TELECOM_3 & Relatório de Sustentabilidade & 2018 \\
\hline TELECOM_4 & Relatório de Administração & 2019 \\
\hline
\end{tabular}

Fonte: Autores (2021)

O corpus de texto foi construído a partir de trechos dos relatórios que descrevem as práticas de QVT, sendo inserido no software IRAMUTEQ v.07 (Interface de $R$ pour les Analyses Multidimensionneles de Textes et de Questionnaires), em arquivo texto na codificação UTF-8 (8bit- Unicode Transformation Format), conforme recomendado por Salviati (2017) (Tabela $1)$.

Tabela 1. Características do corpus de texto inserido no software IRAMUTEQ.

\begin{tabular}{lc}
\hline Número de Textos & 4 \\
Número de Ocorrências & 4938 \\
Número de Palavras (palavras já lematizadas) & 1539 \\
Número de Hapax (palavras que aprecem uma só vez) & 661 \\
Média de Ocorrências por Texto & 1234,50 \\
\hline
\end{tabular}

Fonte: Autores (2021).

Procedeu-se à análise de frequência das palavras (estatística textual clássica ou análise lexicográfica), com a criação do ranking de vocábulos mais prevalentes. Também foi gerada nuvem de palavras, com as formas mais predominantes dispostas no centro da nuvem, circundadas pelos vocábulos em ordem de frequência.

Seguiu-se às análises multivariadas, a começar pela Classificação Hierárquica Descendente (CHD - Método de Reinert) com o intuito de determinar as classes de discursos com seus respectivos vocábulos, representados em um dendograma de classes. Por fim, foi realizada a Análise de Similitude - AS que, apoiada na teoria dos grafos, visou identificar conexões entre vocábulos, representada em uma árvore de similitude.

\section{RESULTADOS}

O corpus textual analisado pelo software IRAMUTEQ foi construído a partir de trechos extraídos dos relatórios integrados e de sustentabilidade disponíveis nos sítios da internet

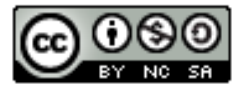


mantidos pelas empresas objeto de estudo; datam dos períodos de 2017 a 2019, selecionadas as versões mais recentes.

Os trechos foram retirados de forma integral das seções pertinentes à "gestão de talentos", "valores e atitudes", "engajamento dos colaboradores", "gestão de pessoas", "comunicação e engajamento", "responsabilidade social" e "recursos humanos".

A lematização do corpus de texto, realizada pelo software, resultou em um total de 138 segmentos de texto (ST), com 4938 ocorrências (palavras), distribuídas em 1539 formas (tipos de palavras) e 963 hapax (palavras mencionadas uma única vez).

Este corpus constitui-se de formas ativas (substantivos ativos, verbos ativos, advérbios e adjetivos) e suplementares (preposições, pronomes, verbos suplementares, substantivos suplementares, artigos, numerais e formas não reconhecidas).

As análises textuais conduzidas por Carvalho et al. (2020) e Fernandes, Silva, Ibiapina \& Silva (2018), utilizando o software IRAMUTEQ, produziram resultados satisfatórios utilizando-se de corpus textuais com menor número de ST, o que nos permite inferir sobre a robustez da análise.

Corrobora para esta percepção o percentual de aproveitamento do corpus pelo software, em $72,46 \%$ (100 ST), superior ao limite recomendado por Mendes et al. (2019).

A Análise Lexicográfica, com a contagem dos vocábulos (palavras) dispostos em uma ordenação crescente (ranking) de frequência, encontra-se demonstrada na Tabela 2.

Tabela 2. Ranking de frequência das palavras no corpus de texto.

\begin{tabular}{c|c|c|c|c|c|c|c}
\hline Ranking & Vocábulo & Frequência & Tipo & Ranking & Vocábulo & Frequência & Tipo $^{\mathbf{1}}$ \\
\hline 1 & colaborador & 48 & nom & 16 & profissional & 14 & adj \\
2 & como & 33 & $\mathrm{adv}$ & 17 & empresa & 14 & nom \\
3 & ao & 31 & $\mathrm{adv}$ & 18 & digital & 14 & adj \\
4 & programa & 30 & nom & 19 & companhia & 14 & nom \\
5 & mais & 25 & $\mathrm{adv}$ & 20 & transformação & 13 & nom \\
6 & novo & 24 & $\mathrm{adj}$ & 21 & trabalhar & 13 & ver \\
7 & ação & 20 & nom & 22 & processo & 13 & nom \\
8 & também & 18 & $\mathrm{adv}$ & 23 & prevenção & 13 & nom \\
9 & saúde & 18 & nom & 24 & segurança & 12 & nom \\
10 & risco & 17 & nom & 25 & meio & 12 & adv \\
11 & desenvolvimento & 17 & nom & 26 & conhecimento & 12 & nom \\
12 & ambiente & 17 & nom & 27 & treinamento & 11 & nom \\
13 & além & 16 & adv & 29 & oportunidade & 11 & nom \\
14 & realizar & 15 & ver & 28 & negócio & 11 & nom \\
15 & promover & 15 & ver & 30 & experiência & 11 & nom \\
\hline
\end{tabular}

${ }^{1}$ nom: substantivo; adv: advérbio; adj: adjetivo; ver: verbo

Fonte: Autores (2021).

Conforme pode ser observado na Tabela 2, tem-se a maior ocorrência na palavra "colaborador" (48 vezes), merecem destaque as palavras "saúde" (18 vezes), "desenvolvimento" (17 vezes) e "risco" (17 vezes), nas 10 primeiras posições do ranking, evidenciando uma ênfase em aspectos ligados à sua saúde, treinamento e segurança do trabalhador. 

telecomunicações brasileiro. Brazilian Journal of Production Engineering, 7(2), 31-47.

Emergem do texto, dispostas na porção intermediária do ranking, as palavras "digital” (14 vezes) e "transformação" (13 vezes), relacionadas às iniciativas de transformação digital em curso nas organizações, as quais impõem desafios com relação à gestão do conhecimento, capacitação e valorização do colaborador, conforme apontado por Sivaraman (2020).

Estas considerações também são sustentadas pela presença das palavras "conhecimento" (12 vezes), "treinamento" (11 vezes), “oportunidade" (11 vezes) e "experiência" (11 vezes), na porção final no ranking.

Nota-se que os aspectos relacionados ao controle de estresse no trabalho, à preservação da saúde física e mental do colaborador e à distribuição de salários, premiações e reconhecimento estão preteridos; tais aspectos são fortemente ligados ao preenchimento das necessidades econômicas, familiares, sociais e de criatividade, apontadas por Sirgy et al. (2001) como pontos relevantes em um processo de avaliação da QVT.

A ausência de tais aspectos revela uma lacuna no conjunto de práticas de QVT nas empresas analisadas, em comparação aos construtos propostos por Mariappanadar (2020), Qamari et al. (2020) e Sierdovski et al. (2020).

O corpus de texto foi classificado conforme a Classificação Hierárquica Descendente (CHD), foi gerado um Dendograma de Classes, demonstrado na Figura 1, com cinco classes de discursos: "Transformação Digital" (Classe 1), "Diversidade na Organização" (Classe 2), "Desenvolvimento do Funcionário" (Classe 3), "Ações de Voluntariado" (Classe 4) e "Saúde e Segurança do Funcionário" (Classe 5). Assim nomeados conforme entendimento do contexto das palavras associadas.

Figura 1. Dendograma de classes.

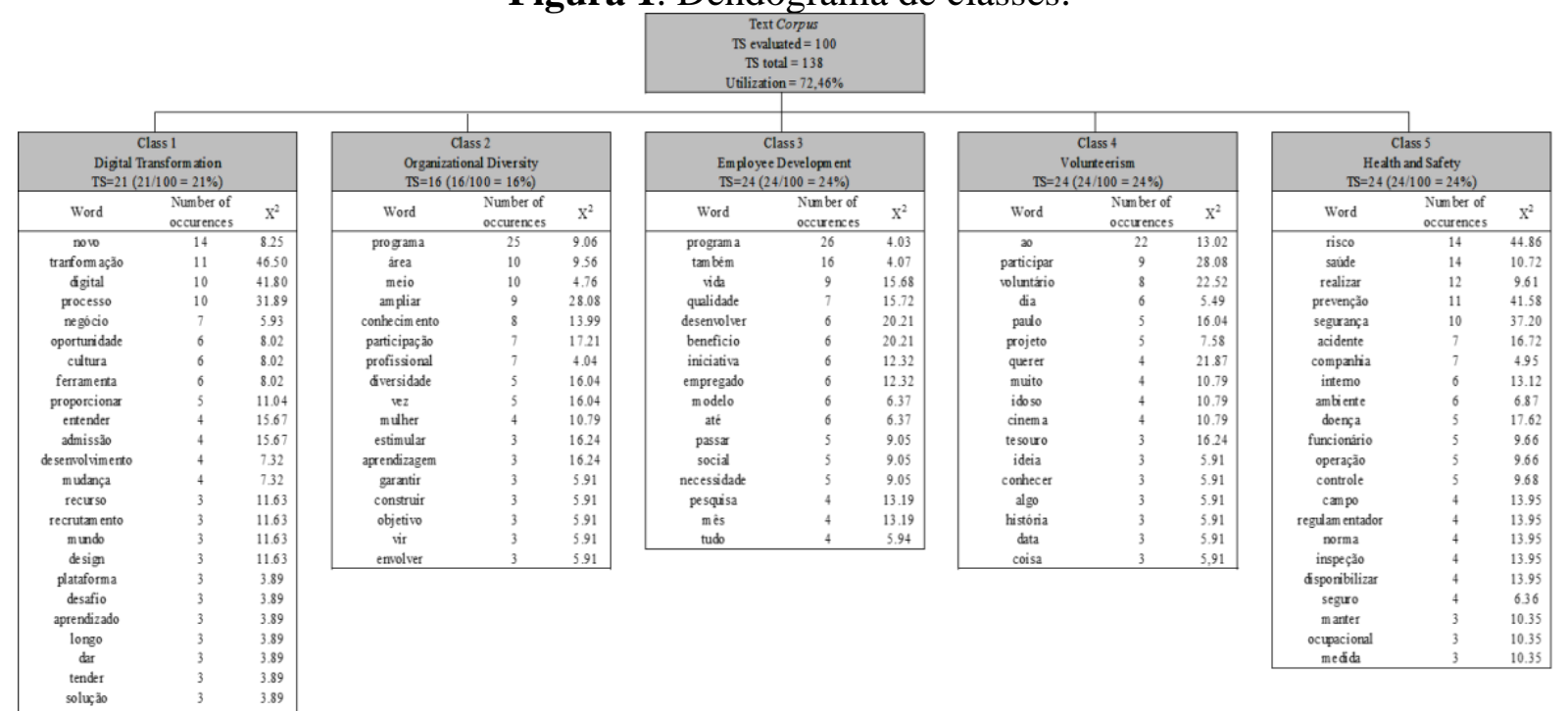

Fonte: Autores (2021).

As classes geradas apresentam a seguinte distribuição: Classe 1, com 21 ST (21\% do total analisado); Classe 2, com 16 ST (16\%); Classe 3, com 24 ST (24\%); Classe 4, com 16 ST $(16 \%)$ e Classe 5, com 23 ST $(23 \%)$. Os vocábulos presentes apresentam X2 de Pearson superior a 3,85, evidenciando a correspondência dos vocábulos com a classe (Camargo \& Justo, 2013; Goulart et al., 2020; Carvalho et al., 2020). 

telecomunicações brasileiro. Brazilian Journal of Production Engineering, 7(2), 31-47.

Como pode ser observado na Figura 1, o Dendograma de Classes demonstra um discurso que enfatiza aspectos ligados aos desafios e oportunidades da transformação digital, à diversidade, desenvolvimento do funcionário, ações de voluntariado e, por fim, a preservação da saúde e segurança do colaborador.

Ao se analisar o percentual de prevalência de cada classe, tem-se a classe "Desenvolvimento do Funcionário" na primeira posição (24\%), seguida pela classe "Saúde e Segurança do Funcionário" (23\%), os vocábulos destas duas classes reforçam o diagnóstico apontado na Análise Lexicográfica, ou seja, uma ênfase em ações de treinamento e desenvolvimento, assim como cuidado na saúde e segurança do colaborador.

A análise dos termos mais associados a cada classe confirma esta percepção, na classe "Desenvolvimento do Funcionário", chamam a atenção os vocábulos "desenvolver" (X2=20,21) e "benefício" (X2=20,21); na classe "Saúde e Segurança do Funcionário", destacam-se os termos "risco" $(\mathrm{X} 2=44,86)$ e "prevenção" $(\mathrm{X} 2=41,38)$.

As demais classes revelam, de forma minoritária, ações de QVT pertinentes a iniciativas de voluntariado (Classe 4) e na construção de uma ambiente de diversidade e participação (Classe 2), a exemplo dos vocábulos "participar" (X2=28,08) e "voluntário" (X2=22,52), na Classe 4; dos vocábulos "ampliar" (X2=28,08), "participação" (X2=17,21), "estimular" $(\mathrm{X} 2=16,24)$ e "diversidade" $(\mathrm{X} 2=16,04)$, na Classe 2.

Percebe-se assim uma deficiência no entendimento do contexto do trabalhador de uma forma mais completa, conforme proposto nos construtos da QVT de Sirgy et al. (2008), Qamari et al. (2020) e Sierdovski et al. (2020), dada ausência de aspectos ligados ao preenchimento das necessidades pessoais.

A Nuvem de Palavras, representada na Figura 2, reforça esta constatação, visto que dispõe no seu centro o vocábulo "colaborador", tendo como próximos os vocábulos "programa", "processo", "segurança", "risco", "saúde” e "desenvolvimento".

Figura 2. Nuvem de palavras por frequência.

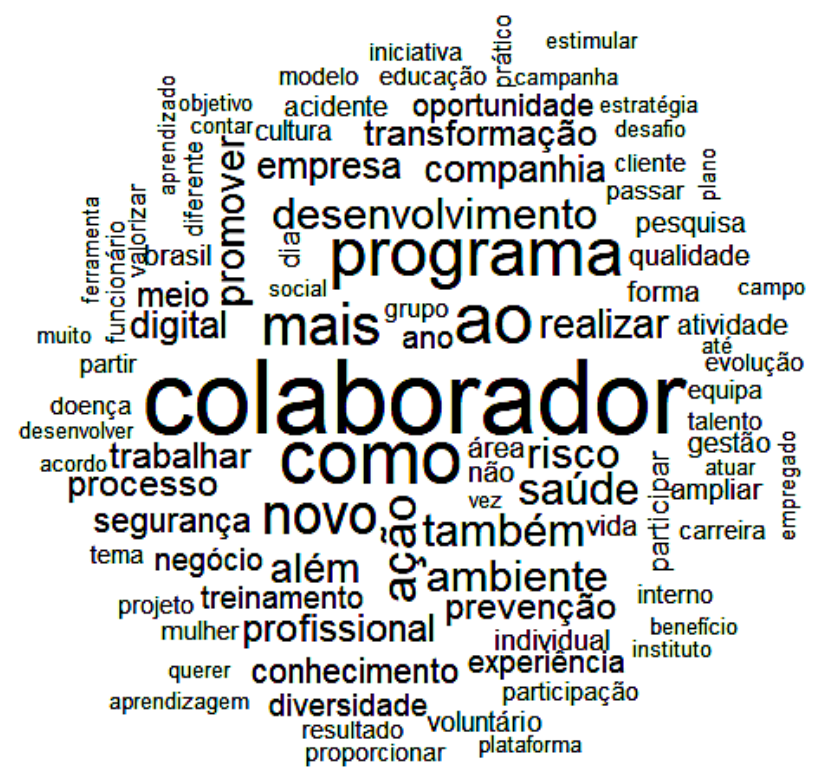

Fonte: Autores (2021). 

telecomunicações brasileiro. Brazilian Journal of Production Engineering, 7(2), 31-47.

A Árvore gerada mediante Análise de Similitude, demonstrada na Figura 3, apresenta 5 ramificações partindo do nó central na palavra "colaborador", da qual partem para os ramos ligados à saúde e segurança, voluntariado, diversidade, desenvolvimento e carreira.

Figura 3. Árvore do corpus de texto, gerada pela Análise de Similitude.

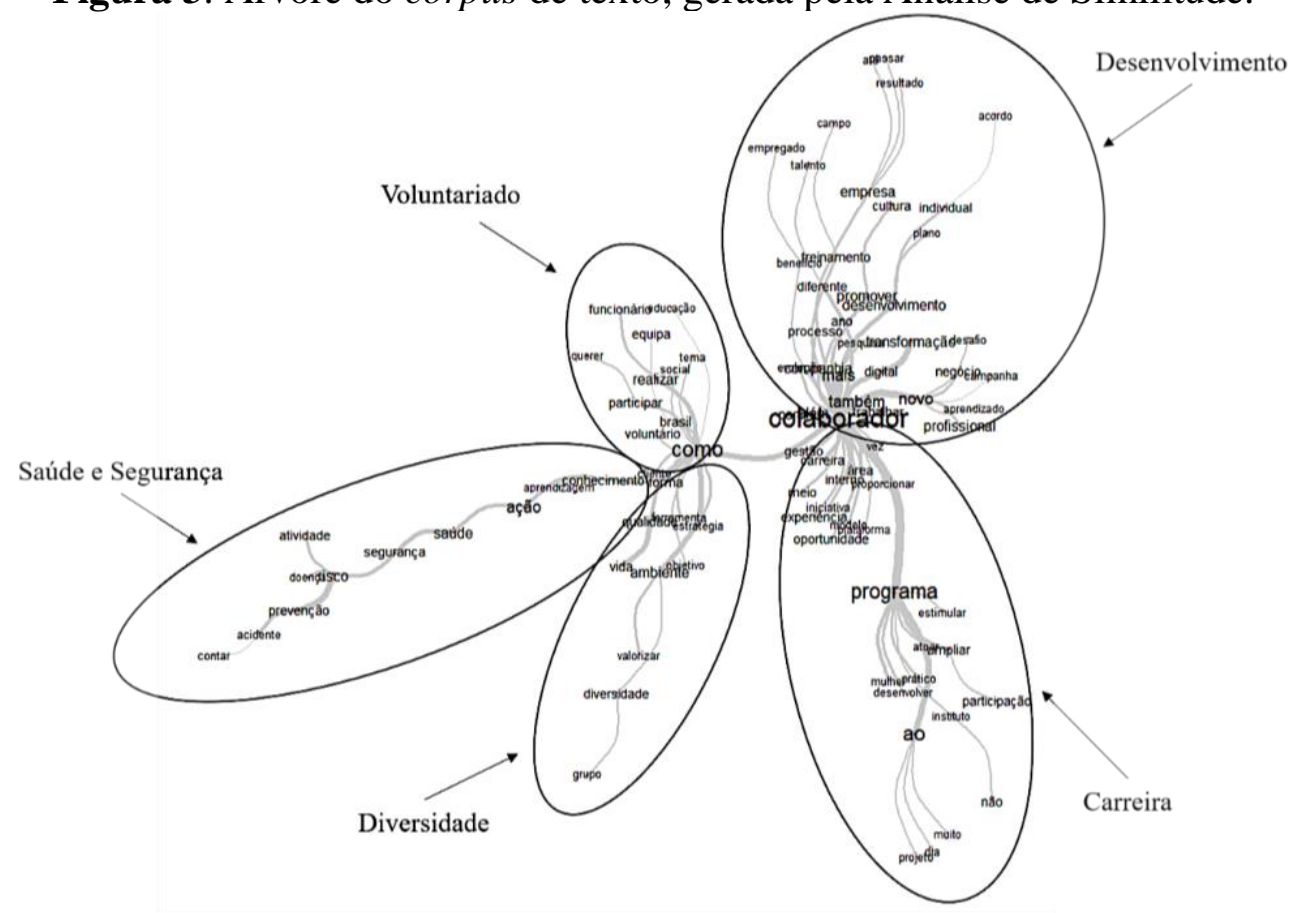

Fonte: Autores (2021).

Desta análise pode-se inferir, em linha com as análises anteriores, que a percepção da QVT compartilhada pelas organizações se inicia no indivíduo, mas enfatiza somente o seu contexto profissional, nas suas questões pertinentes (saúde ocupacional, carreira e desenvolvimento), com uma sutil aproximação às suas necessidades sociais, reveladas pelas iniciativas de voluntariado e desenvolvimento de um ambiente de diversidade.

\section{CONCLUSÕES}

O método de análise textual, pelo uso do software IRAMUTEQ, demonstrou-se eficaz na identificação das práticas de QVT nas empresas estudadas, permitiu a constatação de prevalências e padrões de ações, atendendo ao objetivo deste estudo.

Verifica-se que as organizações estudadas, apesar de terem o colaborador como centro das suas estratégias de QVT, enfatizam práticas que consideram o indivíduo somente no seu contexto profissional.

Esta prevalência se sustenta nos resultados das análises lexicográfica clássica (Ranking de Palavras e Nuvem de Palavras) e multivariada (Classificação Hierárquica Descendente e Análise de Similitude), que sugerem práticas com vínculo à saúde ocupacional, ações de capacitação e desenvolvimento de carreira; associadas a iniciativas de voluntariado e promoção da diversidade.

As práticas identificadas têm forte relação com o preenchimento das necessidades mais elementares de saúde e segurança, seguidas pelas de conhecimento e sociais. Alinham-se aos

\section{@ $\odot \odot \odot$}


indicadores de QVT pertinentes à integração social, condições de trabalho e realização profissional.

Conclui-se que, em resposta às demandas de ordem pessoal do colaborador integrantes do construto da QVT, existe um conjunto de oportunidades de aprimoramento nas práticas das empresas estudadas, particularmente em questões ligadas à redução de estresse e conflitos, as quais tem potencial de contribuir para a manutenção da saúde mental da força de trabalho.

Também se constatam oportunidades de aplicação de práticas de QVT de alto nível como programas de feedback estruturado, mentoria, política de salários, premiações, reconhecimento e maior participação nos processos decisórios, fatores reconhecidos como indutores da motivação e engajamento dos colaboradores.

Este estudo tem limitações no que se refere à generalização dos resultados para outras empresas de telecomunicações, apesar dos quatro grupos estudados representarem mais de 90\% dos acessos do mercado nacional, o setor possui, segundo Agência Nacional de Telecomunicações (ANATEL), 2020b, 80 empresas prestadoras de serviços de TV a cabo, 12 de serviços de telefonia móvel, 48 de telefonia fixa e 5680 de provedoras de internet banda larga.

Como complemento à esta pesquisa, sugere-se que trabalhos futuros examinem as práticas de QVT das empresas analisadas mediante aplicação de questionário com participação em anonimato, a fim de evitar possíveis vieses nas respostas. Esta abordagem metodológica pode fornecer a visão do colaborador, e não institucional (caso deste trabalho) da prática da QVT na organização.

\section{REFERÊNCIAS}

Agência Nacional de Telecomunicações (2019). Relatório Panorama Setorial de Telecomunicações. Brasília: ANATEL, 2019.

Agência Nacional de Telecomunicações (2020). Infográfico Panorama Setorial de Telecomunicações: Maio/2020. Brasília: ANATEL, 2020.

Agência Nacional de Telecomunicações (2020). Painéis de Dados - Ranking das Operadoras. Recuperado em 30 de julho de 2020 de: https://www.anatel.gov.br/paineis/acessos/ranking.

Bansal, P.; Jiang, G. F., \& Jung, J. C. (2015). Managing responsibly in tough economic times: strategic and tactical CSR during the 2008-2009 global recession. Long Range Planning. 48 (1), 69-79. doi: 10.1016/j.lrp.2014.07.002.

Bizzo, W. A., \& Andrade, V. F. (2018). Análise comparativa das normas de gestão de responsabilidade social e sua abrangência. Gestão \& Produção. 25(4), 807-825. doi: 10.1590/0104-530x3866-18.

Bowen, H. R. (1953). Social Responsibilities of Businessman. Iowa City: University of Iowa Press.

Bustamente, M. A., Álvarez, A. J., Villalobos, M. E., \& Lucero, M. I. (2020). Percepción de la calidad de vida laboral de los trabajadores de los centros de salud familiar de la zona central de Chile. Información Tecnológica. 31(3), 65-74. doi:10.4067/S0718-07642020000300065.

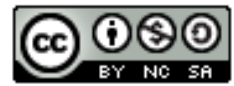



telecomunicações brasileiro. Brazilian Journal of Production Engineering, 7(2), 31-47.

Camargo, B. V., \& Justo, A. M. (2013). Iramuteq: Um Software Gratuito para Análise de Dados Textuais. Temas em Psicologia. 21(2), 513-518. doi: 10.9788/TP2013.2-16.

Carvalho, T. S., Mota, D. M., \& Saab, F. (2020). Utilização do Software IRAMUTEQ na Análise de Contribuições da Sociedade em Processo Regulatório Conduzido pela Agência Nacional de Vigilância Sanitária. Revista Vigilância Sanitária em Debate. 8(1), 10-21. doi: 10.22239/2317-269x.01429.

Damart, S., \& Ledunois, S. A. (2017). Management as an integrating activity - A comparative textual analysis of the work of Mary Parker Follet and Oliver Sheldon. Journal of Management History. 23(4), 452-470. doi:10.1108/JMH-04-2017-0023.

Elkington, J. (1998). Cannibals with forks: The triple bottom line of $21^{\text {st }}$ century business. Environmental Quality Management. 8(1), 37-51. doi: 10.1002/tqem.3310080106.

Fernandes, M. A., Silva, D. R. A., Ibiapina, A. R. S., \& Silva, J. S. (2018). Adoecimento mental e as relações com o trabalho: estudo com trabalhadores portadores de transtorno mental. Revista Brasileira de Medicina do Trabalho. 16(3), 277-286. doi: 10.5327/Z1679443520180110.

Fernández, L. V., Bertin, M. J., \& Pineaur, F. V. (2014). Prácticas de responsabilidad social, reputación corporativa y desempeño financeiro. Revista de Administração de Empresas. 55(3), 329-334. doi: 10.1590/S0034-759020150308.

Goulart, G. S., Viana, M. M., \& Cheung, T. L. (2020). Consumer perception towards familiar and innovative foods: the case of a Brazilian product. British Food Journal. Ahead of print. doi: 10.1108/BFJ-02-2020-0160.

Global Reporting Initiative (2020). About GRI. Recuperado em 02 de junho de 2020 de: https://www.globalreporting.org/information/about-gri/Pages/default.aspx.

Hernandez, M. I. S., Vazquez, D. G., Barcik, A., \& Dziwinski, P. (2016). The Effect of the Internal Side of Social Responsibility on Firm Competitive Success in The Business Services Industry. Sustainability. 8(2), 217-231. doi:10.3390/su8020179.

International Organization for Standardization (1996). ISO 14001: 1996 - Abstract and General Information. Recuperado em 20 de maio de 2020 de: https://www.iso.org/standard/23142.html.

Índice de Sustentabilidade Empresarial (2020). O que é o ISE B3. Recuperado em 03 de junho de 2020 de: http://iseb3.com.br/o-que-e-o-ise.

Jhunior, R. O. S., \& Vilela, N. G. (2018). Sustentabilidade ambiental, econômica e social: ações e práticas de pequenas e médias empresas brasileiras. Organizações \& Sustentabilidade. 6(2), 59-71. doi: 10.5433/2318-9223.2018v6n2p59.

Kim, J.; Milliman, J., \& Lucas, A. (2018). Effects of CSR on employee retention via identification and quality-of-work-life. International Jounal of Contemporary Hospitality Management. 32(3), 1163-1179. doi: 10.1108/IJCHM-06-2019-0573.

Lahlou, S. (2011). Text mining methods: an answer to Chartier e Meunier. Papers on Social Representations. 20(38), 1-7.

Madorran, C., \& Garcia, T. (2014). Corporate social responsibility and financial performance: the spanish case. Revista de Administração de Empresas. 56(1), 20-28. doi: 10.1590/S0034759020160103.

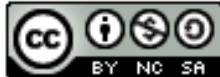


Citação (APA): Agostinho, O. L., Pinto, J. de S., \& Gibbin, R. V. (2021). Análise da qualidade de vida no trabalho no setor de telecomunicações brasileiro. Brazilian Journal of Production Engineering, 7(2), 31-47.

Mascarenhas, A. C. Q., \& Barbosa, A. C. Q. (2019). Gestão de recursos humanos sustentável e responsabilidade socioambiental: uma agenda para debates. Revista de Administração de Empresas. 59(5), 353-364. doi:10.1590/S0034-759020190505.

Mariappanadar, S. (2020). Do HRM systems impose restricitions on empleyee quality of life? Evidence from a sustainable HRM perspective. Journal of Business Research. 118(1), 38-48. doi: 10.1016/j.jbusres.2020.06.039.

Maslow, R. (1943). A theory of human motivation. Psychological Review. 50(4), 370-396.

Mendes, F. R. P., Zangão, M. O. B., Gemito, M. L. G. P., \& Serra, I. C. C. (2016). Representações sociais dos estudantes de enfermagem sobre assistência hospitalar e atenção primária. Revista Brasileira de Enfermagem. 69(2), 343-350. doi: 10.1590/00347167.2016690218i.

Mendes, A. M., Tonin, F. S., Buzzi, M. F., \& Pontarolo, R. (2019). Mapping pharmacy journals: A lexicographic analysis. Research in Social and Administrative Pharmacy. 15(12), 1464-1471. doi: 10.1016/j.sapharm.2019.01.011.

Milne, M. J., Tregidga, H., \& Walton, S. (2003). The triple bottom line: benchmarking New Zealand's early reporters. University of Auckland Business Review. 5(2), 1-14.

Nazir, O., \& Islam, J. U. (2020). Effect of CSR activities on meaningfulness, compassion, and employee engagement: A sense-making theoretical approach. International Journal of Hospitality Management. 90(1), 1-10. doi: 10.1016/j.ijhm.2020.102630.

Orsato, R. J., Garcia, A., Silva, W. M., Simonetti, R., \& Monzoni, M. (2015). Sustainability Indexes: why join in? A study of the 'Corporate Sustainability Index (ISE)' in Brazil. Journal of Cleaner Production. 96(1), 161-170. doi: 10.1016/j.jclepro.2014.10.071.

Prodanov, C. C., \& Freitas, E. C. (2013). Metodologia do Trabalho Científico: Métodos e Técnicas de Pesquisa do Trabalho Acadêmico (2 ${ }^{\mathrm{a}}$ ed.). Novo Hamburgo: Feevale.

Qamari, I. N., Ferdinand, A. T., Dwiatmadja, C., \& Yuniawan, A. (2020). Transformative interaction capability: the mediating role between quality of work life and teamwork performance. International Journal of Quality and Service Sciences. 2(12), 133-148. doi: 10.1108/IJQSS-01-2019-0008.

Robie, G. L. E., Rosini, A. M., \& Oliveira, M. C. (2016). Qualidade de vida no trabalho no contexto da sustentabilidade: Um estudo bibliométrico. Connexio - Revista Científica da Escola de Gestão e Negócios - $e_{\text {Universidade Potiguar. }}$ 2(1).

Salviati, M. E. (2017). Manual do Aplicativo IRAMUTEQ. Recuperado em 09 de julho de 2020 de: http://www.iramuteq.org/documentation/fichiers/manual-do-aplicativo-iramuteqpar-maria-elisabeth-salviati.

Sakar, S., \& Searcy, C. (2016). Zeitgeist os chamaleon? A quantitative analysis of CSR definitions. Journal of Cleaner Production. 135(1), 1423-1435. doi: 10.1016/j.jclepro.2016.06.157.

Shulz, S. A., \& Flanigan, R. L. (2016). Developing competitive advantage using the triple bottom line: a conceptual framework. Journal of Business \& Industrial Marketing. 31(4), 449-458. doi: 10.1108/JBIM-08-2014-0150.

Sierdovski, M., Stefano, S. R., \& Andrade, S. M. (2020). Variáveis de qualidade de vida no trabalho como construto de análise do pilar social da sustentabilidade organizacional: Um 
estudo das práticas nas 150 melhores empresas para se trabalhar no Brasil. Organizações \& Sustentabilidade. 8(1), 24-41. doi: 10.5433/2318-9223.2020v8n1p24.

Singhapakdi, A., Lee, D. J., Sirgy, M. J., \& Senasu, K. (2015). The impact of incongruity between an organization's CSR orientation and its employees' CSR orientation on employee's quality of work life. Journal of Business Research. 68(1), 60-66. doi:10.1016/j.jbusres.2014.05.007.

Sinval, J., Sirgy, M. J., Lee, D., \& Marôco, J. (2019). The Quality of Work Life Scale: Validity Evidence from Brazil and Portugal. Applied Research Quality Life. Ahead of print. doi: 10.1007/s11482-019-09730-3.

Sirgy, M. J., Efraty, D., Siegel, P., \& Lee, D. J. (2001). A New Measure of Quality of Work Life (QWL) based on Need Satisfaction and Spillover Theories. Social Indicators Research. 55(3), 241-302. doi:10.1023/A:1010986923468.

Sirgy, M. J., Reilly, N., Wu, J., \& Efraty, D. (2008). A work-life identity model for wellbeing: Towards a research agenda linking quality of work life programs with quality of life. Applied Reseach Quality Life. 3(3), 181-202. doi:10.1007/s11482-008-9054-6

Sivaraman, A. (2020). Soft side of digital transformation: The connected employee. South Asian Journal of Human Resources Management. 7(1), 121-128. doi: 10.1177/2322093720919336.

Uesangkomsate, P. (2019). Exploring green logistics management in Thai small and mediumsized food exporters. Proceedings of IEEE International Conference on Industrial Engineering and Engineering Management, Macau. 194-198. doi: 10.1109/IEEM44572.2019.8978917

Wardani, L. M. I., \& Anvar, M. S. (2019). The role of quality of work life as mediator: psychological capital and work engagement. Humanities \& Social Sciences Reviews. 7(6), 447-463. doi:10.18510/hssr.2019.7670.

World Comissionon Environmentand Development (1987). Our Common Future Brundtland Comission. Oxford: Oxford University Press. 